\title{
Factores asociados al inicio sexual en adolescentes de ambos sexos de nivel socioeconómico medio-bajo de la Región Metropolitana
}

\author{
Electra González A. ${ }^{1 a}$, Temístocles Molina G. ${ }^{1 b}$, Adela Montero V. ${ }^{1}$, Vania Martínez N. ${ }^{1}$ \\ ${ }^{1}$ Centro de Medicina Reproductiva y Desarrollo Integral del Adolescente. Facultad de Medicina, Universidad de Chile. \\ ${ }^{a}$ Asistente Social. ${ }^{b}$ Bioestadístico.
}

\section{RESUMEN}

Antecedentes: Escasa evidencia está disponible en Chile acerca del inicio de la actividad sexual adolescente. Objetivo: Examinar cuales variables identificadas previamente están asociadas al inicio de la actividad sexual en una población adolescente de nivel socioeconómico medio-bajo de la Región Metropolitana. Método: Los datos corresponden a adolescentes consultantes en CEMERA. Se compararon con adolescentes que habían iniciado actividad sexual y aquellos que no. La información fue recolectada en la primera consulta a 5.854 adolescentes de ambos sexos entre 12 y 19 años. Se usó regresión logística para identificar los factores asociados al inicio sexual. Resultados: $92,6 \%$ mujeres y $7,4 \%$ hombres. El $84,2 \%$ había iniciado actividad sexual y no el $15,8 \%$. Las variables asociadas al inicio sexual fueron: ser hombre; mayor edad; mayor escolaridad; aspiraciones académicas básicas; desempeño escolar bajo; repitencia escolar; no afiliación religiosa; no asistencia a servicios religiosos; tener un trabajo; tener pareja mayor; tener una pareja que trabaje; conversar temas de sexualidad solo con la pareja; más parejas románticas; no participación social; historia de abuso sexual; mayor número de hijos; mala relación padre-hijo; mala comunicación familiar; supervisión parental limitada; historia de paternidad y/o maternidad adolescente en los padres; padres con baja educación; menor supervisión parental. Conclusión: Se necesitan programas que motiven a retardar el inicio sexual. Los programas de educación sexual deberían animar a la conversación de los temas de sexualidad con los padres y capacitar a los educandos a demorar el inicio sexual y/o obtener anticonceptivos, en forma expedita, amigable y confidencial.

\section{PALABRAS CLAVE: Debut sexual, adolescencia, factores de riesgo, diferencias de género}

\section{SUMMARY}

Background: Little evidence is available from Chile concerning adolescent people's sexual debut. Objective: To examine which variables previously identified were associated to the sexual activity in adolescent populations in precarious economic settings of Metropolitan Region. Method: Data from a population of adolescents attending at CEMERA were used to compare those who had sexual debut and those who had not. Analysis used data collected at the adolescent's first visit of 5,854 adolescents, both sexes, aged 12-19. Logistic regression was used to identify factors associated with sexual debut. Results: $92.6 \%$ were females and $7.4 \%$ males. $84.2 \%$ adolescents had sexual debut and $15.8 \%$ did not. Variables associated with sexual debut were: gender (male); age (older); education (higher): low academic aspirations (finishing high school only); low academic performance; repeating an academic year; no religious affiliation; no attendance at religious services; having a job; having an older partner; having a partner who works; discussing topics 
related to sexuality only with partner; more lifetime romantic partners; no social participation; history of sexual abuse; higher number of siblings; poor parent-child relationship; poor family communications; limited parental supervision; history of adolescent parenthood in parents; low parental education; low family involvement in the adolescent's life. Conclusion: Programs that encourage the delay of sexual debut are needed. Sexual health education programs should encourage the discussion of sexuality with parents and enable girls and boys to delay sexual debut or to obtain contraceptives easily and privately.

\section{KEY WORDS: Sexual debut, adolescence, risk factors, gender differences}

\section{INTRODUCCIÓN}

En Chile, del total de nacidos vivos, alrededor del $15 \%$ corresponde a madres menores de 20 años (1). Entre el 2000 y 2005, el número de nacidos vivos declinó consistentemente en todas las edades, pero la tasa de fecundidad adolescente no declinó con la misma intensidad (1). Este problema es especialmente preocupante en adolescentes bajo 15 años, debido a que los nacimientos en adolescentes solteras son generalmente no planeados y a menor edad. Las madres adolescentes solteras están en peores condiciones económicas lo cual aumenta la probabilidad de resultados adversos en el bienestar de la madre e hijo, tanto en el corto como en el largo plazo (2-3). La reducción del embarazo adolescente y de las infecciones de transmisión sexual son metas de las políticas públicas en nuestro país, pero hasta ahora no han tenido los resultados esperados.

En Chile, los adolescentes están iniciando actividad sexual más temprano que en el pasado (3). Sin embargo, a pesar de este hecho, la actividad sexual adolescente no es reconocida por nuestra sociedad. Es escasa la investigación en los factores asociados al inicio de la actividad sexual, lo cual puede dificultar los esfuerzos para proveer servicios que miren las necesidades en salud sexual adolescente (4).

Estudios internacionales han mostrado que el inicio de la actividad sexual está asociados a múltiples factores. Entre los factores personales que se asocian a menor riesgo de inicio sexual, destacan el buen desempeño escolar y la religiosidad (3,511). Mientras que se asocian a inicio sexual más temprano, la influencia de alcohol o drogas (5-7), el antecedente de parejas mayores (12-13), el abuso sexual previo (14-18), la influencia de los pares (1920) y los/as que trabajan (21-22).

En relación a variables familiares, la crianza por ambos padres, el mejor nivel socioeconómico, la mayor comunicación padres-hijo/a, se asocian a la postergación de la actividad sexual (23-24), mien- tras que el pertenecer a familias numerosas puede ser o no un factor de riesgo (25-33). Vivir con ambos padres y la mayor escolaridad de los padres es un factor protector (34-36), mientras que el divorcio o la separación de los padres, los/as adolescentes cuyas madres fueron madres adolescentes, tener padres muy estrictos o muy permisivos (37-39), está asociado al inicio sexual temprano.

El objetivo de este estudio fue examinar las variables que se asocian al inicio sexual en una población adolescente perteneciente al nivel socioeconómico medio-bajo consultante en un centro de atención en salud sexual y reproductiva.

\section{PACIENTES Y MÉTODO}

Diseño de estudio transversal analítico, que analizó los diversos factores previamente identificados en la literatura internacional, como asociados al inicio de la actividad sexual en una población adolescente. La población del estudio fueron adolescentes consultantes en CEMERA de ambos sexos, entre 12 y 19 años que ya habían iniciado actividad sexual. CEMERA es el primer y más importante centro de atención en salud sexual y reproductiva para adolescentes del país, situado en la Región Metropolitana, cuyo principal objetivo es prevenir el primer embarazo. El estudio obtuvo la información de la entrevista estructurada, diseñada para propósitos clínicos y de investigación que aplicó la investigadora principal al ingreso de cada adolescente. A todos los adolescentes se les explicó la modalidad de atención y se les solicitó permiso explícito para utilizar los datos que quedaron registrados en la ficha clínica para fines de investigación, asegurando el resguardo de la confidencialidad y anonimato de los datos. Lo anterior fue formalizado mediante la firma del consentimiento informado especialmente diseñado para estos efectos. El estudio se efectuó en la población de adolescente que consultaron en CEMERA durante el período 1990-2005 (5.854 adolescentes).

Las variables personales analizadas fueron: 
edad a la entrevista, ocupación, escolaridad, desempeño académico (medido por promedio de notas), repitencia de año escolar (fracaso o deserción), aspiraciones académicas, tipo de educación secundaria (científica-humanista, dirigidas a estudiantes que aspiran a estudios universitarios, versus técnica, comercial o industrial, dirigidas a estudiantes que no aspiran a estudios universitarios), participación social (participación en organizaciones deportivas, religiosas, artísticas u otras), edad al inicio de las relaciones románticas, número de relaciones románticas, afiliación religiosa, asistencia a servicios religiosos, con quién conversa temas de sexualidad y antecedentes de abuso sexual.

Las variables familiares analizadas fueron: filiación (vínculo legal entre padres e hijo, matrimonial y no matrimonial), tamaño familia (número de hijos), orden de nacimiento de los hijos, vive actualmente con ambos padres(si/no); figura de crianza (crianza por ambos padres o no), responsable de la supervisión (ambos padres, sólo padre, sólo madre, otro), tipo de permiso durante la semana/fin de semana (muy estricto, estricto, permisivo), calidad relación con padres (definida por autoreporte como buena, regular, mala), comunicación familiar (definida por autoreporte como buena, regular, mala), edad paterna y materna, escolaridad paterna y materna (años de escolaridad cursados), ocupación del padre y madre, antecedente de paternidad adolescente en el padre, antecedente de maternidad adolescente en la madre, funcionamiento familiar (medido por test APGAR familiar) (40), y nivel socio-económico (medido por la escala Graffar) (41).

Se compararon dos grupos: adolescentes que habían iniciado actividad sexual (casos o iniciadores) con aquellos que no habían iniciado actividad sexual (controles o no iniciadores). Dado que la literatura sugiere que hay diferencias en los comportamientos sexuales de hombres y mujeres, las variables fueron estratificadas por sexo.

Análisis estadístico: Los resultados para las variables (discretas y continuas) se expresaron en promedios y porcentajes. En una primera etapa se determinó los adolescentes que habían iniciado actividad sexual. Se realizaron análisis uni y bivariado aplicándose las pruebas estadísticas $\mathrm{Chi}^{2}$ y Test de Diferencia de Medias. Se usó el método de selección Backward para identificar las variables personales y familiares asociadas a la variable dependiente (inicio sexual). Considerando las diferencias en el tamaño de hombres y mujeres, se ajustó un modelo de regresión logística. Este fue adaptado a un modelo de regresión logística múltiple para identificar las variables asociadas al inicio sexual por sexo. Se usó el software STATA 10.0.

\section{RESULTADOS}

Los resultados corresponden a 5.854 adolescentes. El $92,6 \%$ mujeres y $7,4 \%$ hombres. El $84,2 \%$ del total había iniciado actividad sexual. El $90 \%$ son estudiantes y el $94 \%$ pertenece al nivel socioeconómico medio-bajo.

El análisis bivariado mostró que las variables asociadas al inicio sexual fueron: tener un trabajo, bajas aspiraciones académicas (completar solo educación media), y la repitencia para hombres y mujeres. Conversar de sexualidad sólo con la pareja, no participación social, sin afiliación religiosa y no asistencia a servicios religiosos, pareja que trabaja se asoció al inicio sexual sólo en mujeres (no mostrado en Tabla I). Estudiar en un colegio técnico fue asociado al inicio sexual en hombres, y la historia de abuso sexual se asoció al inicio sexual en mujeres (Tabla I). Empleo de la madre fuera del hogar no fue asociado al inicio sexual en hombres o mujeres (no mostrado en Tabla I).

El inicio sexual en ambos sexos fue asociado a la mayor edad, la mayor educación y el mayor número de relaciones románticas. El inicio sexual en mujeres fue asociado a tener pololo mayor y menor promedio de notas. No se relacionó al inicio sexual la edad al momento de la primera relación romántica y los años de escolaridad de la pareja (Tabla II).

Se asoció al inicio sexual en ambos sexos: los permisos limitados para salidas del fin de semana, la baja supervisión parental y una historia de paternidad adolescente en el padre. Se asoció al inicio sexual sólo en mujeres: la ausencia del padre, el no vivir con ambos padres, la mala relación padre-hijo, la mala comunicación familiar e historia de maternidad adolescente en la madre. Se asoció al inicio sexual sólo en los varones: la no supervisión parental en la semana y el tipo de empleo del padre (no mostrado en la Tabla) (Tabla III).

El mayor número de hijos se asoció al inicio sexual en ambos sexos. El menor nivel de escolaridad parental y el pobre funcionamiento familiar se asoció al inicio sexual sólo en las mujeres. Edad de los padres no fue asociada al inicio sexual ni para hombres ni para mujeres (Tabla IV).

El análisis multivariado mostró que, ser hombre, ser mayor, conversar los temas sexuales sólo con pololo o polola, mala comunicación familiar, no asistencia a servicios religiosos fueron asociados al inicio sexual, mientras que mejor promedio de notas fue asociado a postergar actividad sexual (Tabla V). 
Tabla I

VARIABLES PERSONALES CATEGÓRICAS PARA INICIADORES/AS Y NO INICIADORES/AS $(n=5.854)$

\begin{tabular}{|c|c|c|c|c|c|c|}
\hline \multirow[b]{2}{*}{ Variables y categorías } & \multicolumn{2}{|c|}{ Hombres } & \multicolumn{4}{|c|}{ Mujeres } \\
\hline & $\begin{array}{l}\text { Iniciadores } \\
\text { (359) }\end{array}$ & $\begin{array}{c}\text { No } \\
\text { iniciadores } \\
\text { (34) }\end{array}$ & $\begin{array}{c}\text { Valor } \\
p=\end{array}$ & $\begin{array}{l}\text { Iniciadoras } \\
(4.134)\end{array}$ & $\begin{array}{c}\text { No } \\
\text { iniciadoras } \\
(805)\end{array}$ & $\begin{array}{c}\text { Valor } \\
p=\end{array}$ \\
\hline \multicolumn{7}{|l|}{ Actividad: } \\
\hline $\begin{array}{l}\text { Estudiante } \\
\text { Trabajador }{ }^{1}\end{array}$ & $\begin{array}{l}64,3 \\
35,7\end{array}$ & 100 & 0,00 & $\begin{array}{l}89,4 \\
10,6\end{array}$ & $\begin{array}{r}98,5 \\
1,5\end{array}$ & 0,00 \\
\hline \multicolumn{7}{|l|}{ Tipo de enseñanza secundaria: } \\
\hline $\begin{array}{l}\text { Científica-humanística } \\
\text { Técnica, comercial, industrial } \\
\text { Universitaria o superior }\end{array}$ & $\begin{array}{r}46,7 \\
47,7 \\
5,6\end{array}$ & $\begin{array}{r}72,7 \\
21,2 \\
6,1\end{array}$ & 0,00 & $\begin{array}{r}45,5 \\
53,5 \\
1,0\end{array}$ & $\begin{array}{r}47,0 \\
52,3 \\
0,7\end{array}$ & 0,45 \\
\hline \multicolumn{7}{|l|}{ Repitencia escolar: } \\
\hline $\begin{array}{l}\text { No } \\
\text { Sí }\end{array}$ & $\begin{array}{l}60,0 \\
40,0\end{array}$ & $\begin{array}{l}78,8 \\
21,2\end{array}$ & 0,03 & $\begin{array}{l}60,4 \\
39,6\end{array}$ & $\begin{array}{l}72,6 \\
27,4\end{array}$ & 0,00 \\
\hline \multicolumn{7}{|l|}{ Aspiraciones académicas: } \\
\hline $\begin{array}{l}\text { No aspiraciones académicas } \\
\text { Terminar enseñanza media } \\
\text { Universitaria o superior }\end{array}$ & $\begin{array}{r}8,5 \\
26,1 \\
65,4\end{array}$ & $\begin{array}{l}29,4 \\
11,8 \\
58,8\end{array}$ & 0,00 & $\begin{array}{l}13,5 \\
30,1 \\
56,4\end{array}$ & $\begin{array}{c}9,9 \\
19,5 \\
70,6\end{array}$ & 0,00 \\
\hline \multicolumn{7}{|l|}{ Participación social: } \\
\hline $\begin{array}{l}\text { No } \\
\text { Sí }\end{array}$ & $\begin{array}{l}34,2 \\
65,8\end{array}$ & $\begin{array}{l}38,2 \\
61,8\end{array}$ & 0,06 & $\begin{array}{l}64,7 \\
35,3\end{array}$ & $\begin{array}{l}44,9 \\
55,1\end{array}$ & 0,00 \\
\hline \multicolumn{7}{|l|}{ Antecedente de abuso sexual: } \\
\hline $\begin{array}{l}\text { No } \\
\text { Sí }\end{array}$ & $\begin{array}{r}90,9 \\
9,1\end{array}$ & $\begin{array}{r}97,1 \\
2,9\end{array}$ & 0,36 & $\begin{array}{l}84,3 \\
15,7\end{array}$ & $\begin{array}{l}87,3 \\
12,7\end{array}$ & 0,02 \\
\hline \multicolumn{7}{|l|}{ Afiliación religiosa } \\
\hline $\begin{array}{l}\text { No } \\
\text { Sí }\end{array}$ & $\begin{array}{l}26,4 \\
73,6\end{array}$ & $\begin{array}{l}17,7 \\
82,7\end{array}$ & 0,34 & $\begin{array}{l}13,2 \\
86,8\end{array}$ & $\begin{array}{c}9,8 \\
90,2\end{array}$ & 0,05 \\
\hline \multicolumn{7}{|l|}{ Asistencia a servicios religiosos: } \\
\hline $\begin{array}{l}\text { Frecuentemente } \\
\text { Ocasional } \\
\text { Nunca }\end{array}$ & $\begin{array}{l}16,8 \\
21,6 \\
61,5\end{array}$ & $\begin{array}{l}46,7 \\
13,3 \\
40,0\end{array}$ & 0,15 & $\begin{array}{l}22,4 \\
19,3 \\
58,3\end{array}$ & $\begin{array}{l}30,2 \\
24,9 \\
44,9\end{array}$ & 0,00 \\
\hline \multicolumn{7}{|l|}{ Conversa temas sexuales con $^{2}$ : } \\
\hline $\begin{array}{l}\text { Padres } \\
\text { Familiares } \\
\text { Pololo/polola } \\
\text { Amigos } \\
\text { Nadie }\end{array}$ & $\begin{array}{r}33,3 \\
17,6 \\
16,9 \\
23,8 \\
8,2\end{array}$ & $\begin{array}{l}26,5 \\
11,8 \\
11,8 \\
20,6 \\
29,4\end{array}$ & 0,00 & $\begin{array}{l}22,3 \\
12,7 \\
17,5 \\
33,2 \\
14,3\end{array}$ & $\begin{array}{r}38,6 \\
13,7 \\
4,7 \\
30,3 \\
12,7\end{array}$ & 0,00 \\
\hline \multicolumn{7}{|l|}{ Actividad del pololo o polola } \\
\hline $\begin{array}{l}\text { Estudiante } \\
\text { Trabajador }\end{array}$ & $\begin{array}{l}78,5 \\
21,5\end{array}$ & 100 & 0,04 & $\begin{array}{l}45,3 \\
54,7\end{array}$ & $\begin{array}{l}60,6 \\
39,4\end{array}$ & 0,00 \\
\hline
\end{tabular}

\footnotetext{
${ }^{1}$ No asisten más al colegio. ${ }^{2}$ Los mismos sujetos no fueron incluidos en otras categorías. Pololo/a: chilenismo que significa una relación de pareja sin un compromiso mayor.
} 
Tabla II

VARIABLES PERSONALES CONTINUAS PARA INICIADORES/AS Y NO INICIADORES/AS (PROMEDIOS)

\begin{tabular}{|c|c|c|c|c|c|c|}
\hline \multirow[b]{2}{*}{ Variables } & \multicolumn{2}{|c|}{ Hombres } & \multicolumn{4}{|c|}{ Mujeres } \\
\hline & $\begin{array}{l}\text { Iniciadores } \\
\text { (359) }\end{array}$ & $\begin{array}{c}\text { No } \\
\text { iniciadores } \\
(34)\end{array}$ & $\begin{array}{l}\text { Valor } \\
\mathrm{p}=\end{array}$ & $\begin{array}{l}\text { Iniciadoras } \\
(4.134)\end{array}$ & $\begin{array}{c}\text { No } \\
\text { iniciadoras } \\
(805)\end{array}$ & $\begin{array}{c}\text { Valor } \\
p=\end{array}$ \\
\hline Edad (años) & 17,4 & 15,5 & 0,00 & 16,3 & 15,5 & 0,00 \\
\hline Escolaridad (años cursados) & 10,8 & 9,8 & 0,00 & 10,3 & 10,0 & 0,00 \\
\hline $\begin{array}{l}\text { Edad al momento de inicio } \\
\text { relaciones románticas }\end{array}$ & 13,7 & 13,4 & 0,63 & 13,5 & 13,6 & 0,22 \\
\hline Número de relaciones románticas & 4,3 & 2,6 & 0,00 & 3,3 & 2,9 & 0,00 \\
\hline Edad de pololo/polola (años) & 16,1 & 16,2 & 0,94 & 19,5 & 18,4 & 0,00 \\
\hline Escolaridad de pololo/ polola & 10,2 & 10,4 & 0,55 & 11,2 & 11,2 & 0,76 \\
\hline Promedio de notas ${ }^{\star}$ & 5,4 & 5,4 & 0,95 & 5,4 & 5,6 & 0,00 \\
\hline
\end{tabular}

${ }^{\star}$ Escala de notas en Chile va de 1 (mínimo) a 7 (máximo)

Tabla III

VARIABLES FAMILIARES CATEGORICAS PARA INICIADORES/AS Y NO INICIADORES/AS $(n=5.854)$

\begin{tabular}{|c|c|c|c|c|c|c|}
\hline \multirow[b]{2}{*}{ Variables y categorías } & \multicolumn{3}{|c|}{ Hombres } & \multicolumn{2}{|c|}{ Mujeres } & \multirow[b]{2}{*}{$\begin{array}{c}\text { Valor } \\
\mathrm{p}=\end{array}$} \\
\hline & $\begin{array}{l}\text { Iniciadores } \\
\text { (359) }\end{array}$ & $\begin{array}{c}\text { No } \\
\text { iniciadores } \\
\text { (34) }\end{array}$ & $\begin{array}{l}\text { Valor } \\
p=\end{array}$ & $\begin{array}{c}\text { Iniciadoras } \\
(4.134)\end{array}$ & $\begin{array}{c}\text { No } \\
\text { iniciadoras } \\
(805)\end{array}$ & \\
\hline \multicolumn{7}{|l|}{ Figuras de crianza: } \\
\hline Ambos padres & 72,6 & 82,4 & \multirow[t]{2}{*}{0,22} & 66,8 & 71,8 & \multirow[t]{2}{*}{0,00} \\
\hline No ambos padres & 27,4 & 17,6 & & 33,2 & 24,2 & \\
\hline \multicolumn{7}{|l|}{ Relación padre-hijo: } \\
\hline Buena & 60,1 & 55,9 & \multirow[t]{3}{*}{0,06} & 44,6 & 55,0 & \multirow{3}{*}{0,00} \\
\hline Regular & 29,5 & 41,2 & & 38,6 & 36,2 & \\
\hline Mala & 10,4 & 2,9 & & 16,8 & 8,8 & \\
\hline \multicolumn{7}{|l|}{ Vive actualmente con: } \\
\hline Ambos padres & 72,6 & 82,4 & \multirow[t]{2}{*}{0,14} & 66,8 & 71,8 & \multirow{2}{*}{0,00} \\
\hline No ambos padres & 27,3 & 17,6 & & 24,2 & 33,2 & \\
\hline \multicolumn{7}{|l|}{ Comunicación familiar: } \\
\hline Buena & 52,1 & 67,7 & \multirow[t]{3}{*}{0,42} & 41,2 & 53,9 & \multirow[t]{3}{*}{0,00} \\
\hline Regular & 31,5 & 23,5 & & 37,0 & 32,3 & \\
\hline Mala & 16,4 & 8,8 & & 21,8 & 13,8 & \\
\hline \multicolumn{7}{|c|}{ Responsable de la supervisión: } \\
\hline Ambos padres & 31,9 & 35,3 & \multirow[t]{4}{*}{0,05} & 37,7 & 39,2 & \multirow[t]{4}{*}{0,04} \\
\hline Padre & 12,5 & 17,7 & & 11,4 & 9,9 & \\
\hline Madre & 37,5 & 47,0 & & 42,0 & 43,6 & \\
\hline Otro & 18,1 & 0,00 & & 8,9 & 7.2 & \\
\hline \multicolumn{7}{|c|}{ Tipo de permisos durante la semana: } \\
\hline Muy restringido & 27,3 & 52,9 & \multirow[t]{3}{*}{0,01} & 61,8 & 63,8 & \multirow[t]{3}{*}{0,07} \\
\hline Restringido & 46,7 & 41,2 & & 35,2 & 34,4 & \\
\hline Sin restricción & 26,0 & 5,9 & & 3,0 & 1,8 & \\
\hline
\end{tabular}


Tabla III. Continuación.

\begin{tabular}{lcccccc}
\hline & \multicolumn{2}{c}{ Hombres } & & \multicolumn{2}{c}{ Mujeres } \\
Variables y categorías & $\begin{array}{c}\text { No } \\
\text { Iniciadores } \\
(359)\end{array}$ & $\begin{array}{c}\text { No } \\
\text { iniciadores } \\
(34)\end{array}$ & $\begin{array}{c}\text { Valor } \\
\mathrm{p}=\end{array}$ & $\begin{array}{c}\text { Iniciadoras } \\
(4.134)\end{array}$ & $\begin{array}{c}\text { inciadoras } \\
(805)\end{array}$ & $\begin{array}{c}\text { Valor } \\
\mathrm{p}=\end{array}$ \\
\hline Tipo de permisos durante fines de & & & & & & \\
semana: & & & & & & \\
Muy restringido & 5,6 & 29,4 & 0,00 & 10,8 & 19,6 & 0,00 \\
$\begin{array}{l}\text { Restringido } \\
\text { Sin restricción }\end{array}$ & 14,3 & 32,4 & & 28,5 & 33,3 & \\
Antecedente de paternidad adoles- & 80,1 & 38,2 & & 60,7 & 47,1 & \\
cente en el padre: & & & & & & \\
Sí & 16,9 & 2,9 & 0,03 & 16,5 & 12,3 & 0,00 \\
No & 83,1 & 97,1 & & 83,5 & 87,7 & \\
Antecedente de maternidad adoles- & & & & & & \\
cente en la madre: & & & & & & \\
Sí & 37,9 & 32,3 & 0,37 & 41,9 & 35,5 & 0,00 \\
No & 62,1 & 67,7 & & 58,1 & 64,5 & \\
\hline
\end{tabular}

Tabla IV

VARIABLES FAMILIARES CONTINUAS PARA INICIADORES/AS Y NO INICIADORES/AS (PROMEDIOS)

\begin{tabular}{|c|c|c|c|c|c|c|}
\hline \multirow[b]{2}{*}{ Variables } & \multicolumn{3}{|c|}{ Hombres } & \multicolumn{3}{|c|}{ Mujeres } \\
\hline & $\begin{array}{c}\text { Iniciadores } \\
\text { (359) }\end{array}$ & $\begin{array}{c}\text { No } \\
\text { iniciadores } \\
(34)\end{array}$ & $\begin{array}{l}\text { Valor } \\
p=\end{array}$ & $\begin{array}{c}\text { Iniciadoras } \\
(4.134)\end{array}$ & $\begin{array}{c}\text { No } \\
\text { iniciadoras } \\
(805)\end{array}$ & $\begin{array}{c}\text { Valor } \\
p=\end{array}$ \\
\hline Tamaño familia (número de hijos) & 3,3 & 2,8 & 0,00 & 3,3 & 3,0 & 0,00 \\
\hline Orden de nacimiento & 2,1 & 1,9 & 0,23 & 2,2 & 2,0 & 0,00 \\
\hline Edad del padre (años) & 45,0 & 44,2 & 0,56 & 44,2 & 43,7 & 0,09 \\
\hline Edad de la madre (años) & 45,0 & 44,3 & 0,56 & 44,2 & 43,7 & 0,09 \\
\hline Escolaridad del padre & 11,2 & 12,5 & 0,15 & 10,0 & 10,6 & 0,00 \\
\hline Escolaridad de la madre & 10,6 & 10,9 & 0,71 & 9,5 & 10,1 & 0,00 \\
\hline Funcionamiento familiar* & 7,3 & 8,4 & 0,18 & 6,6 & 7,1 & 0,00 \\
\hline
\end{tabular}

*La escala para el APGAR Familiar va de 1 a 10 puntos. 7 a 10 significa familia normo funcional $y<7$ familia disfuncional (40).

Tabla V

MODELO DE REGRESIÓN LOGÍSTICA

(VARIABLES SELECCIONADAS POR SISTEMA BACKWARD)

\begin{tabular}{lccr}
\hline Variable & Odds Ratio & Valor $p=$ & $95 \%$ IC \\
\hline Sexo (hombre) & 3,33 & 0,00 & $1,81-6,14$ \\
Edad (> edad >riesgo) & 1,18 & 0,00 & $1,09-1,29$ \\
Promedio de notas (> promedio < riesgo) & 0,62 & 0,00 & $0,51-0,76$ \\
Conversar temas de sexualidad solo con pololo/polola & 2,32 & 0,00 & $1,52-3,54$ \\
Mala comunicación familiar & 1,51 & 0,01 & $1,12-2,04$ \\
No asistencia a servicios religiosos & 1,57 & 0,00 & $1,27-1,94$ \\
Edad del pololo/polola (> edad > riesgo) & 1,09 & 0,00 & $1,05-1,14$ \\
\hline
\end{tabular}

Pololo/a: chilenismo que significa una relación de pareja sin un compromiso mayor. 
Dada la diferencia entre el número de hombres y mujeres (mujeres $92,4 \%$ y hombres $7,6 \%$ ) y usando variables seleccionadas, se aplicó un modelo de regresión logística, los resultados son similares excepto en la no asistencia a servicios religiosos $(p<0,05)$. Los resultados de este modelo mostraron que el ser hombre aumentó el riesgo de inicio sexual en 3,35 veces; por cada año de edad el riesgo de inicio sexual aumenta 1,19 veces, y el mejor promedio de notas fue un factor protector, disminuyendo el riesgo 0,58 veces.

Las adolescentes que conversan temas de sexualidad sólo con sus pololos, están 2,24 veces más en riesgo de inicio sexual que las que conversan con otras personas. Las adolescentes con mala comunicación familiar tienen 1,96 veces más riesgo de inicio sexual que las con buena comunicación familiar. Por cada año de diferencia en la edad del pololo el riesgo de inicio aumenta 1,09 veces (Tabla VI).
Los resultados del modelo estratificado para el sexo femenino se presenta en la Tabla VIII. Mostró que el riesgo de inicio sexual aumenta 1,18 veces por cada año de edad; el promedio de notas alto disminuye el riesgo 0,63 veces; las que conversan temas de sexualidad solo con el pololo enfrentan un riesgo 2,33 veces mayor de inicio sexual que las que conversan con otras personas; la mala comunicación familiar aumenta el riesgo de inicio sexual 1,47 veces; las que no asisten a servicios religiosos tienen 1,62 veces más riesgo de inicio sexual que quienes asisten regularmente; por cada año de diferencia que tiene la adolescente con su pololo el riesgo de inicio sexual aumenta 1,09 veces. Ninguna de las variables analizadas en mujeres (Tabla VII) fue significativa cuando se estratificó por sexo masculino (Tabla VIII).

Tabla VI

MODELO DE REGRESIÓN LOGÍSTICA (PESO DE LA POBLACIÓN)

\begin{tabular}{lccc}
\hline Variable & Odds Ratio & Valor $\mathrm{p}=$ & $95 \%$ IC \\
\hline Sexo (hombre) & 3,35 & 0,00 & $1,76-6,35$ \\
Edad (> edad > riesgo) & 1,19 & 0,02 & $1,03-1,39$ \\
Promedio de notas (> promedio < riesgo) & 0,58 & 0,03 & $0,36-0,94$ \\
Conversa temas de sexualidad solo con pololo/polola & 2,24 & 0,05 & $1,01-4,94$ \\
Mala comunicación familiar & 1,96 & 0,00 & $1,42-2,71$ \\
No asistencia a servicios religiosos & 1,24 & 0,27 & $0,85-1,80$ \\
Edad pololo/polola (> edad > riesgo) & 1,09 & 0,005 & $1,02-1,15$
\end{tabular}

Pololo/a: chilenismo que significa una relación de pareja sin un compromiso mayor.

Tabla VII

MODELO DE REGRESIÓN LOGÍSTICA (MUJERES)

\begin{tabular}{lccc}
\hline Variable & Odds Ratio & Valor $\mathrm{p}=$ & $95 \% \mathrm{C} \mathrm{I}$ \\
\hline Edad (> edad > riesgo) & 1,18 & 0,00 & $1,09-1,28$ \\
Promedio de notas (> promedio < riesgo) & 0,63 & 0,00 & $0,51-0,77$ \\
Conversa temas de sexualidad solo con pololo & 2,33 & 0,00 & $1,51-3,61$ \\
Mala comunicación familiar & 1,47 & 0,01 & $1,09-1,99$ \\
No asistencia a servicios religiosos & 1,62 & 0,00 & $1,30-2,01$ \\
Edad pololo (> edad > riesgo) & 1,09 & 0,00 & $1,05-1-15$ \\
\hline
\end{tabular}

Pololo/a: chilenismo que significa una relación de pareja sin un compromiso mayor. 
Tabla VIII

MODELO DE REGRESIÓN LOGÍSTICA (HOMBRES)

\begin{tabular}{lccc}
\hline Variable & Odds Ratio & Valor $\mathrm{p}=$ & $95 \% \mathrm{C} \mathrm{I}$ \\
\hline Edad & 1,22 & 0,32 & $0,82-1,83$ \\
Promedio de notas & 0,49 & 0,40 & $0,09-2,59$ \\
Conversa temas de sexualidad solo con polola & 2,15 & 0,46 & $0,28-16,56$ \\
No asistencia a servicios religiosos & 0,66 & 0,53 & $0,18-2,38$ \\
Edad de la polola & 0,99 & 0,95 & $0,69-1,42$ \\
\hline
\end{tabular}

Pololo/a: chilenismo que significa una relación de pareja sin un compromiso mayor.

\section{DISCUSIÓN}

El objetivo de este estudio fue estudiar las variables que se asocian al inicio sexual en una población adolescente perteneciente al nivel socioeconómico medio-bajo consultante en un centro de atención en salud sexual y reproductiva. Los resultados más relevantes de este estudio muestran que las variables asociadas con el inicio sexual fueron: ser hombre, mayor edad, mayor escolaridad, aspiraciones académicas básicas (sólo terminar enseñanza media), desempeño escolar bajo, repitencia escolar, no afiliación religiosa, no asistencia a servicios religiosos, tener un trabajo, tener pareja mayor, tener una pareja que trabaje, conversar temas de sexualidad solo con la pareja, más parejas románticas, no participación social, historia de abuso sexual, mayor número de hijos, mala relación padre-hijo/a, mala comunicación familiar, supervisión parental limitada, historia de paternidad y/o maternidad adolescente en los padres, baja educación en los padres, y menor supervisión parental. A continuación analizaremos algunos de estos resultados comparándolos con estudios efectuados en países desarrollados.

En Chile, como también acontece en muchos países desarrollados, los adolescentes están iniciando actividad sexual a edades más tempranas que en el pasado (3). Sin embargo, a pesar de este hecho, la actividad sexual adolescente no es reconocida o aceptada abiertamente por nuestra sociedad, lo cual puede dificultar los esfuerzos para proveer servicios integrales que miren las reales necesidades en salud adolescente (1-4).

El análisis de las variables personales asociados al inicio sexual muestra que concordante con los estudios en países desarrollados (3-8), el buen desempeño escolar y la religiosidad, independiente de la religión que profesaban los adolescentes, se asoció a menor riesgo de inicio precoz de debut sexual. Mientras que adolescentes de mayor edad, mayor escolaridad y adolescentes mujeres con parejas románticas mayores (pololos) y que trabajan, mostraron un mayor riesgo (5-13).

Concordante con lo reportado por la literatura, el antecedente de abuso sexual se asoció al inicio sexual en las mujeres del estudio. Este antecedente expone a las mujeres a mayor riesgo en sus comportamientos sexuales: inicio sexual más temprano, relaciones de pareja con extraños, mas parejas sexuales y a fracasar en el uso de anticonceptivos (14-18).

Los adolescentes que trabajan tienen menos supervisión parental y por lo tanto más oportunidades de ser expuestos a la influencia de los pares que sostienen valores diferentes a los de los padres (19-22), esta asociación fue demostrada en el presente estudio.

La crianza por ambos padres, independiente si los padres son o no casados, se asoció a la postergación de la actividad sexual sólo en las mujeres y no en ambos sexos como muestra la literatura (23-24). También nuestros resultados son concordantes con los estudios que muestran que los adolescentes que viven en familias numerosas están en mayor riesgo de experimentar actividad sexual, ya que el mayor número de hijos puede limitar el tiempo para monitorear su comportamiento, especialmente cuando estos son adolescentes (25-29). Sin embargo, hay autores que sostienen que los padres pueden guiar directa o indirectamente a sus hijos transmitiendo estándares de comportamiento durante el proceso de socialización, por lo tanto, los padres que mantienen actitudes restrictivas hacia la sexualidad de sus hijos, aunque tengan varios hijos, pueden organizar sus actividades de tal manera que los puedan supervisar o controlar adecuadamente (30-33).

La mala comunicación entre padres e hijos se asoció al riesgo de debut sexual precoz sólo en las 
mujeres, contrario a lo que muestran los estudios internacionales (34-36). Coincidente con lo reportado en la literatura, se observó que las adolescentes cuyas madres fueron madres adolescentes, estuvieron en mayor riesgo de inicio sexual precoz. Esto se explicaría porque ven a sus madres como más permisivas acerca de la actividad sexual antes del matrimonio, independiente de las reales creencias sostenidas por sus progenitoras $(37,38)$. Un hallazgo importante de este estudio, no reportado en la literatura, fue que el tener padre con antecedente de paternidad adolescente fue un factor de riesgo del inicio sexual para hombres y mujeres. Hay consenso en que vivir con ambos padres es un factor protector, mientras que el divorcio o separación de ellos, especialmente cuando es la madre quien asume la custodia y manutención de los hijos, es un factor de riesgo de debut sexual precoz (34-36).

Mayor escolaridad parental se asoció a la postergación del inicio de la actividad sexual sólo en las mujeres. Esto puede ser explicado porque los padres con mayor educación tienen más altas aspiraciones académicas para las hijas, y pueden tener más habilidades parentales para desalentar el inicio sexual en las hijas que en los hijos. Es importante destacar que los adolescentes que percibían a sus padres como muy permisivos en los permisos de fin de semana, tuvieron más riesgo de inicio sexual precoz, a diferencia de los estudios que muestran que los adolescentes que ven a sus padres como muy restrictivos o muy permisivos tienen más riesgo de inicio sexual comparados con aquellos que perciben a sus padres como moderadamente estrictos $(23,27)$.

En relación al empleo de la madre fuera del hogar, que la literatura reporta como factor de riesgo (37), en nuestro estudio este antecedente no fue un factor de riesgo. En nuestro medio tradicionalmente son las madres quienes supervisan el comportamiento de los hijos, y durante su jornada laboral que puede ser muy extensa (más de 8 horas fuera del hogar), los hijos tienen mayor oportunidad para tener encuentros sexuales, incluso en el mismo hogar, pese a lo anterior, esta situación no se asoció con riesgo de precocidad en el debut sexual.

La fortaleza de este estudio es que es el primero que reporta los factores asociados al inicio sexual en adolescentes chilenos de nivel socioeconómico medio-bajo, sin embargo, las limitaciones de este estudio es que los resultados son aplicables solo a esos adolescentes y no pueden ser generalizados a la población adolescente de otros estratos socioeconómicos. A pesar de esto, los resultados deberían ser considerados en intervenciones destinadas a prevenir el inicio sexual en una población vulnerable y en la necesidad de intervenciones de políticas públicas. La pobreza, las condiciones económicas inseguras y las bajas expectativas académicas, desaniman a las adolescentes a alcanzar la movilidad social a través de logros en educación y así posponer la actividad sexual y las consecuencias de un embarazo temprano (36-39).

\section{CONCLUSIÓN}

Intervenciones tempranas para motivar a los adolescentes a demorar el inicio sexual deben considerar múltiples niveles y diversas estrategias, que deben estar centradas en las necesidades de los adolescentes y en los factores tanto personales como familiares que influyen el inicio sexual.

\section{REFERENCIAS}

1. De Bruyn M. Jóvenes en riesgo: Los adolescentes y la salud sexual, Agenda Salud, 2001;23:1-8. Isis Internacional, Santiago, Chile

2. Molina R, Molina T, González E. Madres niñas-adolescentes de 14 años y menos: Un grave problema de salud pública no resuelto en Chile. Rev Med Chile 2007;135:79-86.

3. González E, Molina T, Montero A, Martínez V. Comportamientos sexuales y diferencias de género en adolescentes usuarios de un sistema público de salud universitario. Rev Med Chile 2007;135:1261-69.

4. González E, Molina T, Montero A, Martínez V, Leyton C. Factores asociados a la continuidad del uso anticonceptivo en adolescentes solteras nulíparas. Rev Med Chile 2009;137:1187-92.

5. O'Donnell L, Donnell RC, Stueve A. early sexual initiation and subsequent sex-related risks among urban minority youth: the reach for health study. Fam Plann Perspect 2001;33:268-75

6. Hofferth S. Factors affecting initiation of sexual intercourse. In: Risking the future: Adolescent sexuality, pregnancy and childbearing, Washington, DC: National Academy Press 1987;1:7-35

7. SERNAM, Análisis de las conductas de las/os adolescentes Región Metropolitana, Santiago, Chile, 1997, No. 53

8. Molina R, Sandoval J. González E. (Eds.) Salud Sexual y Reproductiva en la Adolescencia, 2003. 1a Edición. Editorial Mediterráneo, pp 415-560, caps 29-40.

9. Forste R, Heator TB. Initiation of sexual activity among female adolescents, Youth Soc 1988;19:250-68.

10. Miller B, Olson TD, Sexual attitudes and behavior of high school students in relation to background and contextual factors. J Sex Research 1988;24:194-200.

11. Thornton A, Camburn D. Religious participation and adolescent sexual behavior. J Marriage Fam 1989;51:641-53.

12. Kris L, Gowen S, Feldman S, Diaz R, Donovan SI. A comparison of the sexual behavior and attitudes of adolescent girls with older vs similar-aged boyfriends. J Youth Adolesc 2004;33:167 
13. Marin VB, Coyle KK, Gomez C, Carvajal CS, Kirby $\mathrm{BD}$. Older boyfriends and girlfriends increase risk of sexual initiation in young adolescents. J Adolesc Health 2000;27:409-18.

14. Raj A, Silverman JG, Amaro H. The relationship between sexual abuse and sexual risk among high school students. Findings from the 1997 Massachusetts youth risk behavior. Matern Child Health J 2000;4:125-34.

15. Roosa WM, Jenn-Yun T, Reinholtz C, Angelini JP. The relationship of childhood sexual abuse to teenage pregnancy. J Marriage Fam 1997;59:119-30.

16. Boyer D, Fine D. Sexual abuse as factor in adolescent pregnancy and child maltreatment. Fam Plann Perspect 1992;24:11-9.

17. Fergusson DM, Horwood J, Lynshy MT. Childhood sexual abuse, adolescent sexual behavior and sexual revictimization. Child Abuse Negl 1997;21:789-803.

18. Buzi SR, Tortolero RS, Roberts ER. The impact of a history of sexual abuse in high-risk sexual behaviors among females attending alternative schools. Adolescence 2003;38:595-611.

19. Miller CB, McCoy JK, Olson TD. Dating age and stage as correlates of adolescent attitudes and behavior. $J$ Adolescent Research 1986;1:361-71.

20. Thornton A. The courtship process and adolescent sexuality. J Family Issues 1990;11:239-73.

21. Jorgensen S, King SL, Torrey BA. Dyadic and social network influences on adolescent exposure to pregnancy risk. J Marriage Fam 1980;42:141-55.

22. Newcomer S, Gilbert M, Udry JR. Perceived and actual same sex peer behavior as determinants of adolescent sexual behavior. Paper presented at the Annual Meeting of the American Psychological Association. In: Adolescent sexual behavior, Pregnancy and Parenting: Research through the 1980s. J Marriage Fam 1990;1025-44.

23. Thornton A, Camburn D. The influence of the family on premarital sexual attitudes and behavior. Demography 1987;24:323-40.

24. Miller CB, Moore K. Adolescent sexual behavior, pregnancy and parenting: Research through the 1980s. J Marriage Fam 1990;1025-44.

25. McBride MV. Black adolescent females: A comparison of early versus late coital initiators. Family Relations 1994;43:342-48.

26. Lammers C, Ireland M, Resnick M, Blum R. Influences on adolescents' decision to postpone onset of sexual intercourse: A survival analysis of virginity among youths aged 13 to 18 years. J Adolesc Health 2000;26:42-8.

27. Hoogan PD, Kitawua ME. The impact of social status, family structure, and neighborhood on the fertility of black adolescents. Am J Sociology 1985;90:825-55.

28. Mueller KE, Powers WG. Parent-child sexual discussion: Perceived communicator style and subsequent behavior. Adolescence 2000;25:469-82.

29. Fox Greer L, Inazu JK. Patterns and outcomes of mother-daughter communication about sexuality. J Social Issues 1980;36:7-29.

30. Montenegro AH. Educación sexual de niños y adolescentes. Rev Med Chile 2000;128:571-3.

31. Fernández FL, Bustos ML, González WL. Creencias, actitudes y conocimientos en educación sexual. Rev Med Chile 2000;128:574-83.

32. Miller C B, Moore K. Adolescent sexual behavior, pregnancy and parenting: Research through the 1980s. J Marriage Fam 1990;1025-44.

33. Miller SK, Forehand R, Kotchick B. Adolescent sexual behavior in two ethnic minors samples: The role of family variables. J Marriage Fam 1999;61:85-98.

34. Miller B, Sneesby KR. Educational correlates of adolescent's sexual attitudes and behavior. J Youth Adolesc 1988;17:521-30.

35. Kahn J, Smith K, Roberts E. Family communication and adolescent sexual behavior. American Institute for Research. Cambridge. MA, 1984.

36. O'Connell M, Rogers C. Out-of-wedlock births, premarital pregnancies and their effect on family formation and dissolution. Fam Plann Perspect 1984;16:157-62.

37. Newcomer S, Udry JR. Mothers' influence on the sexual behavior of their teenage children. J Marriage Fam 1984;46:477-85.

38. Newcomer S, Udry JR. Parental marital effects on adolescent sexual behavior. J Marriage Fam 1987;49:235-40.

39. Forste $R$, Heaton TB. Initiation of sexual activity among female adolescents. Youth \& Society 1988;19:250-68.

40. Smilkstein G. The Family APGAR: A proposal for family function test and its use by physicians. J Family Practice 1978;6:1231-9.

41. Álvarez ML, Muzzo S, Ivanovic D. Escala para medición del nivel socioeconómico en el área de salud. Rev Med Chile 1985;113:243-49. 